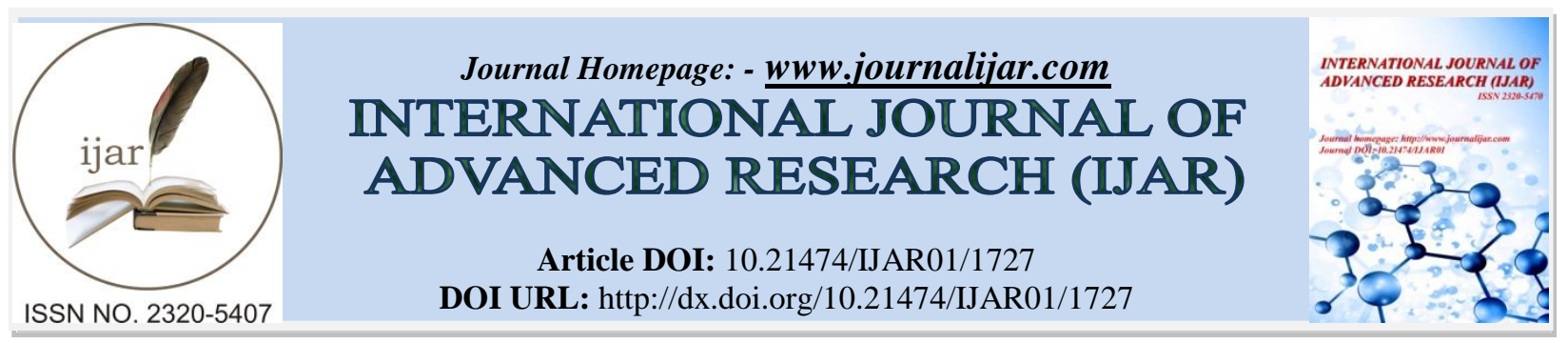

RESEARCH ARTICLE

\title{
Knowledge and Awareness of Metabolic Syndrome Relevant Conditions among College Students in University of Tabuk, KSA.
}

\author{
Marwa A. Abd Elazeem ${ }^{1,2}$, Latifa M. Al Anzi ${ }^{3 *}$, Amnah A. Al Anazi ${ }^{3}$ \\ 1. Department of Pathology, Faculty of Medicine, Tanta University, Tanta, Egypt. \\ 2. Department of Pathology, Faculty of Medicine, University of Tabuk, Tabuk, Saudi Arabia. \\ 3. Students in Faculty of Medicine, University of Tabuk, Tabuk, Saudi Arabia.
}

\section{Manuscript Info}

\section{Manuscript History}

Received: 16 July 2016

Final Accepted: 16 August 2016

Published: September 2016

Key words:-

Metabolic syndrome, knowledge, diabetes, cardiovascular, obesity, atherosclerosis.

\section{Abstract}

Background: Metabolic syndrome (MS) is a major public-health and clinical challenge worldwide, it has gained increasing interest due to its association with type II diabetes mellitus and increased risk of cardiovascular diseases as well as causing mortality.

Aim: Is to assess knowledgeand awareness of MSand conditions associated with it among a sample of college students in the University of Tabuk to design health care promotion schemes for improving MS knowledge and awareness in the community.

Materials and Methods: An online questionnaire was administered to 97college students of the University of Tabuk. The questionnaire includes questions related to six conditions: diabetes, hypertension, obesity, serum cholesterol level, atherosclerosis and stroke. Students' answers were scored and statistically analyzed.

Results: Most of students could correctly recognized symptoms and complications of diabetes, hypertension, serum cholesterol level and obesity. Lower scores were observed in the students' knowledge about manifestations, complications and treatment of atherosclerosis and stroke. There was a significant statistical relationship between students' knowledge about MS relevant conditions and students' education major $(\mathrm{p}<0.05)$.

Conclusion: Students' knowledge and awareness about conditions relevant to MS can be improved especially among non-medical college students in order to promote a healthy lifestyle and to prevent metabolic syndrome relevant conditions complications.

Copy Right, IJAR, 2016,. All rights reserved.

\section{Introduction:-}

Metabolic syndrome (MS) is a major public-health problem worldwide as consequences of urbanization, consumption of fatty diet, increasing obesity, and sedentary life habits [1]. The WHO definition in 1998 was the first to tie together the key components of insulin resistance: obesity, dyslipidemia and hypertension. The definition mandates that insulin resistance must be present; without it, even if all the other criteria were met, the patient would not have metabolic syndrome [2]. 
According to the National Cholesterol Education Program's Adult Treatment Panel III (NCEP ATP III) report in 2005, MS is identified in individuals when at least three of the following five risk factors are present simultaneously: increased waist circumference, elevated blood pressure, elevated serum triglyceride, reduced HDL cholesterol (HDL-C) and elevated fasting plasma glucose [3]. The underlying cause of this syndrome is not yet known, but its manifestation has been linked to obesity (especially abdominal obesity) and insulin resistance [4]. Worldwide prevalence of MS ranges from $<10 \%$ to as much as $84 \%$, depending on the region, urban or rural environment, composition (sex, age and race) of the studied population [5,6]. In general, the international diabetes federation estimates that one-quarter of the world's adult population has MS [7].

Studies on the prevalence of MS among Arab populations are limited, but the available data suggest that it is an increasingly a common problem [8-10]. In Saudi Arabia the first study was published in 2002, and included patients with diabetes treated at King Abdulaziz University Hospital in Jeddah. The study reported the prevalence of MS which was $56 \%$ in men and $57 \%$ in women, with hypertension being the most common component [11].

MS increases the risk of type 2 diabetes mellitus by about 5-fold and the risk of developing cardiovascular disease (CVD) by about 2-fold over 5 to 10 years [12]. Further, patients with the MS are at 2- to 4-fold increased risk of stroke, a 3- to 4-fold increased risk of myocardial infarction, and 2-fold the risk of dying from MS relevant conditions compared with those without the syndrome [13]. Thus, early risk factor detection and intervention is essential to prevent and reduce the development of MS and, eventually, cardiovascular health problems later on [2].

In this context, college students should be aware of MS relevant conditions hence appropriate lifestyle choices can be made. However, a lack of awareness and knowledge might hinder such a change. Therefore, students who are unaware of MS or lack knowledge about MS may not perceive themselves at risk for this condition and, consequently, may go undiagnosed until cardiovascular and diabetes complications occur [2].

Thus, the aim of this study is to assess knowledge and awareness of MS relevant conditions among a sample of college students in University of Tabuk. As the outcomes of this study may have implications for designing health promotion programs based on students' knowledge to improve students' and community health.

\section{Material and Methods:-}

A cross-sectional study was carried on 97 medical and non-medical college students (60 females, 37 males) to assess the knowledge and awareness of MS among them, University of Tabuk, during the period from April 2015-January 2016. The questions about knowledge and awareness of MS relevant conditions was collected through a designed online questionnaire including 34 questions: 6 questions related to their knowledge regarding diabetes mellitus, 6 questions about symptoms, risk factors and complications of hypertension, 6 questions related to hazards and complication of obesity, 4 questions about serum cholesterol level, 7 questions about the effect and complications of arteriosclerosis and 5 questions related to risk factors, manifestations and relation of stroke to diabetes [2]. The response options to the questions were "Yes", "No", or "Do not know".

\section{The MS questionnaire:}

\begin{tabular}{|c|c|c|}
\hline Diabetes & $\begin{array}{l}\dot{1} \\
: \\
: \\
: \\
:\end{array}$ & $\begin{array}{l}\text { There are different types of diabetes. } \\
\text { Pregnant women have a reduced risk of acquiring diabetes } \\
\text { Eye disorders can be consequences of diabetes } \\
\text { Hereditary factors play a major role in the development of diabetes in all types } \\
\text { Poor appetite is a frequent symptom of diabetes. } \\
\text { Frequent urination is a classic symptom of diabetes. }\end{array}$ \\
\hline Hypertension & $\begin{array}{l}\dot{1} \\
: \\
: \\
: \\
:\end{array}$ & $\begin{array}{l}\text { Hypertension can cause dizziness. } \\
\text { Hypertension is associated with heredity. } \\
\text { Medication can usually be discontinued after hypertension has lowered. } \\
\text { Individuals with hypertension are less likely to suffer from atherosclerosis. } \\
\text { Hypertension can cause renal damage. } \\
\text { Hypertension can lead to eye disorders. }\end{array}$ \\
\hline Obesity & $\cdot$ & $\begin{array}{l}\text { Obese individuals have an elevated risk of myocardial infarction. } \\
\text { Obese individuals have an elevated risk of atherosclerosis. }\end{array}$ \\
\hline
\end{tabular}




\begin{tabular}{|c|c|}
\hline & 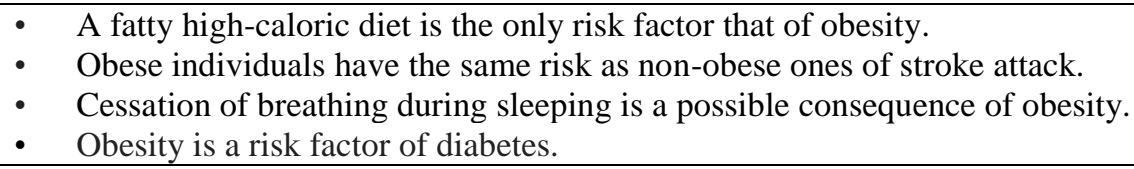 \\
\hline High serum cholesterol & $\begin{array}{l}\text { - High serum cholesterol causes arteriosclerosis } \\
\text { - } \quad \text { High serum cholesterol is treated with medication only. } \\
\text { - } \quad \text { A low cholesterol diet can add in therapy for high serum cholesterol. } \\
\text { - } \quad \text { Diabetes usually associated with high serum cholesterol level. }\end{array}$ \\
\hline Atherosclerosis & 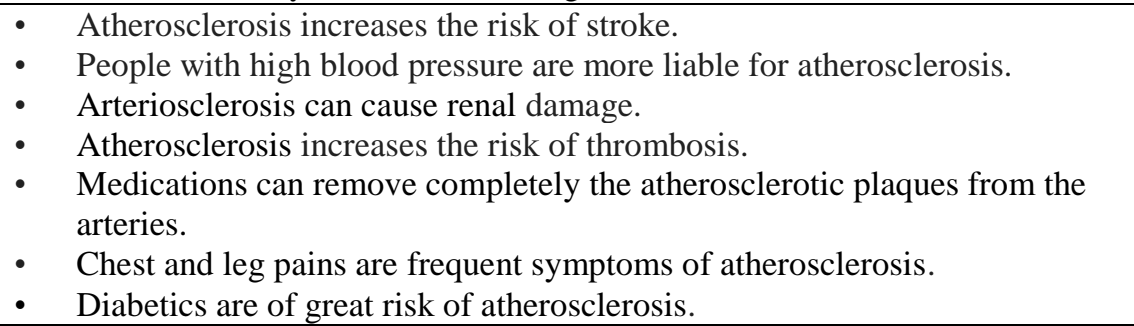 \\
\hline Stroke & $\begin{array}{l}\text { - } \quad \text { Individuals with diabetes are more liable to suffer from a stroke. } \\
\text { - } \quad \text { Stroke is associated with difficulty in moving arms and legs. } \\
\text { - } \quad \text { Permanent speech defects are possible consequences of stroke. } \\
\text { - } \quad \text { A stroke is often followed by memory disturbances. } \\
\text { - Hypertension is a major risk factor of stroke. }\end{array}$ \\
\hline
\end{tabular}

\section{Statistical analysis:-}

Data were statistically analyzed using SPSS (Statistical Package for Social Sciences version 20, IBM, Armonk, NY, USA). Mean and standard deviation (SD) were calculated for continuous variables and frequencies and percentages for categorical variables. Chi-square $(\chi 2)$ test was used to determine the significance of association between students' knowledge and their age group, gender and education group (medical or non-medical). The results were considered as statistically significant if the $\mathrm{P}$ value was less than 0.05 .

\section{Results:-}

\section{People Demographics:-}

A total sample of 97 college students in the University of Tabuk had completed the questionnaire survey, 37 students were males and 60 were females $(38.1 \%$ and $61.8 \%$ respectively). The age of the students ranged between 16-25 years, with a mean of years (20.28 \pm 2.7$) .62$ students were in medical colleges (63.9\%) and most of them were last years of education and the remaining participating students were in non-medical colleges (36.1\%) as shown in

Table 1: Demographic data of respondents

\begin{tabular}{|l|c|c|}
\hline & Number & Percent \\
\hline Age & $20.28 \pm 2.7$ & \\
Mean \pm SD & 47 & $48.5 \%$ \\
$16-20$ & 50 & $51.5 \%$ \\
$21-25$ & & $38.1 \%$ \\
\hline Gender & 37 & $61.8 \%$ \\
Male & 60 & $63.9 \%$ \\
Female & & $36.1 \%$ \\
\hline College & 62 & 35 \\
Medical & & \\
Non-medical & & \\
\hline
\end{tabular}

Knowledge and awareness of conditions associated with metabolic syndrome among students Diabetes mellitus knowledge and awareness:-

Out of total 97 respondents, $83(85.6 \%)$ could correctly answered that there are several types of diabetes, most of them were females $(88.3 \%)$ and of medical students $(96.8 \%)$. Also, the majority of students could recognize that diabetes can cause eye disorders and frequent urination, most of them was of older age group $(72 \%$ and $88 \%$ respectively) and in medical colleges $(77.4 \%$ and $80.6 \%)$. While only 42 students $(43.3 \%)$ were be able to answer 
that hereditary factors play a major role in the development of diabetes in all types, also 53 (54.6\%) students could correctly answered that poor appetite is a frequent symptom of diabetes correctly. There was a statistical significant difference between medical and non-medical students regarding their knowledge of diabetes $(p=0.04)$, whereas no statistical significant difference was found between the age and gender of the students in relation to their answers ( $\mathrm{p}=0.22$ and 0.5 respectively). Table 2

Table 2: Questions related to diabetes mellitus knowledge answered correctly by the respondents

\begin{tabular}{|c|c|c|c|c|c|c|c|}
\hline & \multicolumn{2}{|c|}{ Age } & \multicolumn{2}{|c|}{ Gender } & \multicolumn{2}{|c|}{ Education } & \multirow{2}{*}{$\begin{array}{c}\text { Total } \\
\text { No } \\
(\%)\end{array}$} \\
\hline & $\begin{array}{c}16-20 \\
\text { No } \\
(\%)\end{array}$ & $\begin{array}{l}21- \\
25 \\
\text { No } \\
(\%)\end{array}$ & $\begin{array}{l}\text { Male } \\
\text { No } \\
(\%)\end{array}$ & $\begin{array}{c}\text { Female } \\
\text { No } \\
(\%)\end{array}$ & $\begin{array}{c}\text { Medical } \\
\text { No } \\
(\%)\end{array}$ & $\begin{array}{c}\text { Non- } \\
\text { medical } \\
\text { No }(\%)\end{array}$ & \\
\hline There are different types of diabetes & $38(80.9)$ & $\begin{array}{c}45 \\
(90)\end{array}$ & $\begin{array}{c}30 \\
(81.1)\end{array}$ & $\begin{array}{c}53 \\
(88.3)\end{array}$ & $\begin{array}{c}60 \\
(96.8)\end{array}$ & $\begin{array}{c}23 \\
(65.7)\end{array}$ & $\begin{array}{c}83 \\
(85.6)\end{array}$ \\
\hline $\begin{array}{l}\text { Pregnant women have a reduced risk of } \\
\text { acquiring diabetes }\end{array}$ & $\begin{array}{l}31 \\
(66)\end{array}$ & $\begin{array}{c}39 \\
(78)\end{array}$ & $\begin{array}{c}28 \\
(75.7)\end{array}$ & $\begin{array}{c}42 \\
(70)\end{array}$ & $\begin{array}{c}50 \\
(80.6)\end{array}$ & $\begin{array}{c}20 \\
(57.1)\end{array}$ & $\begin{array}{c}70 \\
(72.2)\end{array}$ \\
\hline $\begin{array}{l}\text { Eye disorders can be consequences of } \\
\text { diabetes }\end{array}$ & $27(57.4)$ & $\begin{array}{c}36 \\
(72) \\
\end{array}$ & $\begin{array}{c}24 \\
(64.9) \\
\end{array}$ & $\begin{array}{c}39 \\
(65) \\
\end{array}$ & $\begin{array}{c}48 \\
(77.4) \\
\end{array}$ & $\begin{array}{c}15 \\
(42.9)\end{array}$ & $\begin{array}{c}63 \\
(64.9) \\
\end{array}$ \\
\hline $\begin{array}{l}\text { Hereditary factors play a major role in the } \\
\text { development of diabetes in all types }\end{array}$ & $13(27.7)$ & $\begin{array}{c}29 \\
(58)\end{array}$ & $\begin{array}{c}17 \\
(45.9)\end{array}$ & $\begin{array}{c}25 \\
(41.7)\end{array}$ & $\begin{array}{c}30 \\
(48.4)\end{array}$ & $\begin{array}{c}12 \\
(34.3)\end{array}$ & $\begin{array}{c}42 \\
(43.3)\end{array}$ \\
\hline $\begin{array}{l}\text { Poor appetite is a frequent symptom of } \\
\text { diabetes }\end{array}$ & $22(46.8)$ & $\begin{array}{c}31 \\
(62)\end{array}$ & $\begin{array}{c}23 \\
(62.2)\end{array}$ & $\begin{array}{c}30 \\
(50)\end{array}$ & $\begin{array}{c}38 \\
(61.3)\end{array}$ & $\begin{array}{c}15 \\
(42.9)\end{array}$ & $\begin{array}{c}53 \\
(54.6)\end{array}$ \\
\hline $\begin{array}{l}\text { Frequent urination is a classic symptom of } \\
\text { diabetes }\end{array}$ & $40(85.1)$ & $\begin{array}{c}44 \\
(88)\end{array}$ & $\begin{array}{c}30 \\
(81.1)\end{array}$ & $\begin{array}{l}54 \\
(90)\end{array}$ & $\begin{array}{c}50 \\
(80.6)\end{array}$ & $\begin{array}{c}34 \\
(97.1)\end{array}$ & $\begin{array}{c}84 \\
(86.6)\end{array}$ \\
\hline $\begin{array}{l}\text { Chi-square } \\
\mathrm{P} \text { value }\end{array}$ & $\begin{array}{l}2.86 \\
0.22\end{array}$ & & & & & $\begin{array}{l}7.582 \\
0.04^{*}\end{array}$ & \\
\hline
\end{tabular}

\section{Hypertension knowledge and awareness:-}

Most of the students were able to answer correctly the questions related to symptoms and complications of hypertension. 80 students $(82.5 \%)$ knew that hypertension can cause dizziness; most of them were of older age group (92\%), females (88.3\%), and medical students $(93.5 \%)$. Similarly, the majority of the responders could realize that hypertension is associated with heredity factors $(60.8 \%)$, medication should not discontinued after hypertension has lowered corrected (66\%), hypertension can be cause renal damage $(75.3 \%)$, and hypertension can lead to eye disorders $(68 \%)$. However, only $56.7 \%$ of students were able to identify that individuals with hypertension are more likely to suffer from arteriosclerosis (56.7\%).a statistical significant relation was found between the age and education of students ( $\mathrm{p}=0.04$ and 0.03 respectively) and their knowledge, while no significant difference was found in relation to gender $(\mathrm{p}=0.31)$. Table 3 
Table 3: Questions related to hypertension knowledge answered correctly by the respondents

\begin{tabular}{|c|c|c|c|c|c|c|c|}
\hline & \multicolumn{2}{|c|}{ Age } & \multicolumn{2}{|c|}{ Gender } & \multicolumn{2}{|c|}{ Education } & \multirow{2}{*}{$\begin{array}{l}\text { Total } \\
\text { No } \\
(\%)\end{array}$} \\
\hline & $\begin{array}{c}16-20 \\
\text { No } \\
(\%)\end{array}$ & $\begin{array}{c}21-25 \\
\text { No }(\%)\end{array}$ & $\begin{array}{l}\text { Male } \\
\text { No } \\
(\%)\end{array}$ & $\begin{array}{c}\text { Female } \\
\text { No } \\
(\%)\end{array}$ & $\begin{array}{l}\text { Medical } \\
\text { No } \\
(\%)\end{array}$ & $\begin{array}{l}\text { Non-medical } \\
\text { No }(\%)\end{array}$ & \\
\hline $\begin{array}{l}\text { Hypertension can cause } \\
\text { dizziness }\end{array}$ & $\begin{array}{c}34 \\
(72.3)\end{array}$ & $\begin{array}{c}46 \\
(92) \\
\end{array}$ & $\begin{array}{c}27 \\
(73)\end{array}$ & $\begin{array}{c}53 \\
(88.3)\end{array}$ & $\begin{array}{c}58 \\
(93.5) \\
\end{array}$ & $\begin{array}{c}22 \\
(62.9) \\
\end{array}$ & $\begin{array}{c}80 \\
(82.5) \\
\end{array}$ \\
\hline $\begin{array}{l}\text { Hypertension is } \\
\text { associated with heredity } \\
\text { factors }\end{array}$ & $\begin{array}{c}21 \\
(44.7)\end{array}$ & $\begin{array}{l}38 \\
(76)\end{array}$ & $\begin{array}{c}22 \\
(59.5)\end{array}$ & $\begin{array}{c}37 \\
(61.7)\end{array}$ & $\begin{array}{c}45 \\
(72.6)\end{array}$ & $\begin{array}{c}14 \\
(40)\end{array}$ & $\begin{array}{c}59 \\
(57.7)\end{array}$ \\
\hline $\begin{array}{l}\text { Medication can usually } \\
\text { be discontinued after } \\
\text { hypertension has } \\
\text { lowered }\end{array}$ & $\begin{array}{c}24 \\
(51.1)\end{array}$ & $\begin{array}{c}40 \\
(80)\end{array}$ & $\begin{array}{c}21 \\
(56.8)\end{array}$ & $\begin{array}{c}43 \\
(71.7)\end{array}$ & $\begin{array}{c}45 \\
(72.6)\end{array}$ & $\begin{array}{c}19 \\
(54.3)\end{array}$ & $\begin{array}{c}64 \\
(66)\end{array}$ \\
\hline $\begin{array}{l}\text { Individuals with } \\
\text { hypertension are less } \\
\text { likely to suffer from } \\
\text { arteriosclerosis }\end{array}$ & $\begin{array}{c}19 \\
(40.4)\end{array}$ & $\begin{array}{l}36 \\
(72)\end{array}$ & $\begin{array}{c}25 \\
(67.6)\end{array}$ & $\begin{array}{c}30 \\
(50)\end{array}$ & $\begin{array}{c}42 \\
(67.7)\end{array}$ & $\begin{array}{c}13 \\
(37.1)\end{array}$ & $\begin{array}{c}55 \\
(56.7)\end{array}$ \\
\hline $\begin{array}{l}\text { Hypertension can cause } \\
\text { renal damage }\end{array}$ & $\begin{array}{c}31 \\
(66)\end{array}$ & $\begin{array}{c}42 \\
(84)\end{array}$ & $\begin{array}{c}24 \\
(64.9)\end{array}$ & $\begin{array}{c}49 \\
(81.7)\end{array}$ & $\begin{array}{c}50 \\
(80.6)\end{array}$ & $\begin{array}{c}23 \\
(65.7)\end{array}$ & $\begin{array}{c}73 \\
(75.3)\end{array}$ \\
\hline $\begin{array}{l}\text { Hypertension can lead } \\
\text { to eyedisorders }\end{array}$ & $\begin{array}{c}29 \\
(61.7) \\
\end{array}$ & $\begin{array}{c}37 \\
(74) \\
\end{array}$ & $\begin{array}{c}26 \\
(70.3) \\
\end{array}$ & $\begin{array}{c}40 \\
(66.7) \\
\end{array}$ & $\begin{array}{c}46 \\
(74.2) \\
\end{array}$ & $\begin{array}{c}18 \\
(51.4) \\
\end{array}$ & $\begin{array}{c}64 \\
(66) \\
\end{array}$ \\
\hline $\begin{array}{l}\text { Chi-square } \\
\mathrm{P} \text { value }\end{array}$ & & & & & & $\begin{array}{l}7.632 \\
0.03^{*} \\
\end{array}$ & \\
\hline
\end{tabular}

Awareness and knowledge of the students about obesity:-

Most of the students whether medical and non-medical have good knowledge of obesity risk factors and complications. In which $84.5 \%$ of the students knew that obese individuals have an elevated risk of myocardial infarction, $86.6 \%$ could identify obesity as risk factor of atherosclerosis and $70 \%$ of the students realized that obese individuals have not the same risk as non-obese ones of a stroke attack. However, only 58.8\% of the responders showed good knowledge regarding fatty high-caloric is the only risk factor of obesity. No significant statistical difference was found between students' knowledge about obesity related conditions and their age, gender or education ( $\mathrm{p}=0.16,0.34$ and 0.31 respectively). Table 4

Table 4: Questions related to obesity knowledge answered correctly by the respondents

\begin{tabular}{|c|c|c|c|c|c|c|c|}
\hline & \multicolumn{2}{|c|}{ Age } & \multicolumn{2}{|c|}{ Gender } & \multicolumn{2}{|c|}{ Education } & \multirow{2}{*}{$\begin{array}{l}\text { Total } \\
\text { No } \\
(\%)\end{array}$} \\
\hline & $\begin{array}{l}16-20 \\
\text { No } \\
(\%)\end{array}$ & $\begin{array}{c}21-25 \\
\text { No }(\%)\end{array}$ & $\begin{array}{l}\text { Male } \\
\text { No } \\
(\%)\end{array}$ & $\begin{array}{c}\text { Female } \\
\text { No } \\
(\%)\end{array}$ & $\begin{array}{l}\text { Medical } \\
\text { No } \\
(\%)\end{array}$ & $\begin{array}{c}\text { Non-medical } \\
\text { No }(\%)\end{array}$ & \\
\hline $\begin{array}{l}\text { Obese individuals have } \\
\text { an elevated risk of } \\
\text { myocardial infarction }\end{array}$ & $\begin{array}{c}38 \\
(80.9) \\
\end{array}$ & $\begin{array}{c}44 \\
(88) \\
\end{array}$ & $\begin{array}{c}33 \\
(89.2)\end{array}$ & $\begin{array}{c}49 \\
(81.7) \\
\end{array}$ & $\begin{array}{c}48 \\
(77.4)\end{array}$ & $\begin{array}{c}34 \\
(97.1) \\
\end{array}$ & $\begin{array}{c}82 \\
(84.5) \\
\end{array}$ \\
\hline $\begin{array}{l}\text { Obese individuals have } \\
\text { an elevated risk of } \\
\text { atherosclerosis }\end{array}$ & $\begin{array}{c}39 \\
(83)\end{array}$ & $\begin{array}{c}45 \\
(90)\end{array}$ & $\begin{array}{c}30 \\
(81.1)\end{array}$ & $\begin{array}{c}54 \\
(90)\end{array}$ & $\begin{array}{c}50 \\
(80.6)\end{array}$ & $\begin{array}{c}34 \\
(97.1)\end{array}$ & $\begin{array}{c}84 \\
(86.6)\end{array}$ \\
\hline $\begin{array}{l}\text { A fatty high-caloric diet } \\
\text { is the only risk factor of } \\
\text { obesity }\end{array}$ & $\begin{array}{c}24 \\
(51.1)\end{array}$ & $\begin{array}{c}33 \\
(66)\end{array}$ & $\begin{array}{c}21 \\
(56.8)\end{array}$ & $\begin{array}{c}36 \\
(60)\end{array}$ & $\begin{array}{c}40 \\
(64.5)\end{array}$ & $\begin{array}{c}17 \\
(48.6)\end{array}$ & $\begin{array}{c}57 \\
(58.8)\end{array}$ \\
\hline $\begin{array}{l}\text { Obese individuals have } \\
\text { the same risk as non- } \\
\text { obese ones of a stroke } \\
\text { attack }\end{array}$ & $\begin{array}{c}28 \\
(59.6)\end{array}$ & $\begin{array}{l}40 \\
(80)\end{array}$ & $\begin{array}{c}23 \\
(62.3)\end{array}$ & $\begin{array}{l}45 \\
(75)\end{array}$ & $\begin{array}{c}44 \\
(71)\end{array}$ & $\begin{array}{c}24 \\
(68.6)\end{array}$ & $\begin{array}{c}68 \\
(70.1)\end{array}$ \\
\hline $\begin{array}{l}\text { Cessation of breathing } \\
\text { during sleeping is a } \\
\text { possible consequence of }\end{array}$ & $\begin{array}{c}33 \\
(70.2)\end{array}$ & $\begin{array}{c}46 \\
(92)\end{array}$ & $\begin{array}{c}29 \\
(78.4)\end{array}$ & $\begin{array}{c}50 \\
(83.3) \\
\end{array}$ & $\begin{array}{c}47 \\
(75.9)\end{array}$ & $\begin{array}{c}32 \\
(91.4)\end{array}$ & $\begin{array}{c}79 \\
(81.4)\end{array}$ \\
\hline
\end{tabular}




\begin{tabular}{|l|c|c|c|c|c|c|c|}
\hline obesity & & & & & \\
\hline Obesity is a risk factor & 39 & 46 & 28 & 57 & 54 & 31 & 85 \\
of diabetes & $(83)$ & $(92)$ & $(75.7)$ & $(95)$ & $(87.1)$ & $(88.6)$ & $(87.6)$ \\
\hline Chi-square & \multicolumn{2}{|c|}{3.076} & \multicolumn{2}{|c|}{2.119} & & 2.994 \\
P value & \multicolumn{2}{|c|}{0.16} & 0.34 & & 0.31 \\
\hline
\end{tabular}

Awareness and knowledge of the students about serum cholesterol level

All questions related to serum cholesterol level were answered correctly by most of the responders where $74.2 \%$ of students recognized that high serum cholesterol causes arteriosclerosis, $73.2 \%$ correctly answered both that high serum cholesterol is not treated with medication only and diabetes usually associated with high serum cholesterol level. $92.8 \%$ of students knew that a low cholesterol diet can add in therapy for high serum cholesterol. A statistical significant difference was found between education of the students and their knowledge $(p=0.02)$, whereas no statistical significance difference between age or gender and students' knowledge about cholesterol level associated conditions ( $\mathrm{p}=0.319$ and 0.41 respectively). Table 5

Table 5: Questions related to serum cholesterol level knowledge answered correctly by the respondents

\begin{tabular}{|c|c|c|c|c|c|c|c|}
\hline & \multicolumn{2}{|c|}{ Age } & \multicolumn{2}{|c|}{ Gender } & \multicolumn{2}{|c|}{ Education } & \multirow{2}{*}{$\begin{array}{l}\text { Total } \\
\text { No } \\
(\%)\end{array}$} \\
\hline & $\begin{array}{l}16-20 \\
\text { No } \\
(\%)\end{array}$ & $\begin{array}{c}21-25 \\
\text { No }(\%)\end{array}$ & $\begin{array}{l}\text { Male } \\
\text { No } \\
(\%)\end{array}$ & $\begin{array}{l}\text { Female } \\
\text { No } \\
(\%)\end{array}$ & $\begin{array}{l}\text { Medical } \\
\text { No } \\
(\%)\end{array}$ & $\begin{array}{l}\text { Non-medical } \\
\text { No }(\%)\end{array}$ & \\
\hline $\begin{array}{l}\text { High serum cholesterol } \\
\text { causes arteriosclerosis }\end{array}$ & $\begin{array}{c}34 \\
(72.3) \\
\end{array}$ & $\begin{array}{c}38 \\
(76)\end{array}$ & $\begin{array}{c}30 \\
(81.1)\end{array}$ & $\begin{array}{c}42 \\
(70)\end{array}$ & $\begin{array}{c}51 \\
(82.3) \\
\end{array}$ & $\begin{array}{c}21 \\
(60)\end{array}$ & $\begin{array}{c}72 \\
(74.2)\end{array}$ \\
\hline $\begin{array}{l}\text { High serum cholesterol } \\
\text { is treated with } \\
\text { medication only }\end{array}$ & $\begin{array}{c}31 \\
(66) \\
\end{array}$ & $\begin{array}{c}40 \\
(80) \\
\end{array}$ & $\begin{array}{c}27 \\
(73) \\
\end{array}$ & $\begin{array}{c}44 \\
(73.3) \\
\end{array}$ & $\begin{array}{c}50 \\
(80.6) \\
\end{array}$ & $\begin{array}{c}21 \\
(60)\end{array}$ & $\begin{array}{c}71 \\
(73.2) \\
\end{array}$ \\
\hline $\begin{array}{l}\text { A low cholesterol diet } \\
\text { can add in therapy for } \\
\text { high serum cholesterol }\end{array}$ & $\begin{array}{c}42 \\
(89.4) \\
\end{array}$ & $\begin{array}{c}48 \\
(96) \\
\end{array}$ & $\begin{array}{c}33 \\
(89.2) \\
\end{array}$ & $\begin{array}{c}57 \\
(95) \\
\end{array}$ & $\begin{array}{c}60 \\
(96.8) \\
\end{array}$ & $\begin{array}{c}30 \\
(85.7) \\
\end{array}$ & $\begin{array}{c}90 \\
(92.8) \\
\end{array}$ \\
\hline $\begin{array}{l}\text { Diabetes usually } \\
\text { associated with high } \\
\text { serum cholesterol level }\end{array}$ & $\begin{array}{c}28 \\
(59.6) \\
\end{array}$ & $\begin{array}{r}43 \\
(86) \\
\end{array}$ & $\begin{array}{c}30 \\
(81.1)\end{array}$ & $\begin{array}{c}41 \\
(68.3) \\
\end{array}$ & $\begin{array}{c}54 \\
(87.1) \\
\end{array}$ & $\begin{array}{c}17 \\
(48.6)\end{array}$ & $\begin{array}{c}71 \\
(73.2) \\
\end{array}$ \\
\hline $\begin{array}{l}\text { Chi-square } \\
\text { P value }\end{array}$ & & & & & & $\begin{array}{l}7.916 \\
0.02^{*}\end{array}$ & \\
\hline
\end{tabular}

Awareness and knowledge of the students about atherosclerosis:-

Most students showed fair good knowledge about atherosclerosis manifestations and complications and most of them were medical students, while the majority of non- medical students showed poor knowledge about atherosclerosis risk and complications. There was a statistical difference between students' knowledge and their age, gender and education $(\mathrm{p}=0.002,0.0001$ and 0.0001 respectively). Table 6 
Table 6: Questions related to atherosclerosis level knowledge answered correctly by the respondents

\begin{tabular}{|c|c|c|c|c|c|c|c|}
\hline & \multicolumn{2}{|c|}{ Age } & \multicolumn{2}{|c|}{ Gender } & \multicolumn{2}{|c|}{ Education } & \multirow{2}{*}{$\begin{array}{c}\text { Total } \\
\text { No } \\
(\%) \\
\end{array}$} \\
\hline & $\begin{array}{c}16-20 \\
\text { No }(\%)\end{array}$ & $\begin{array}{c}21-25 \\
\text { No }(\%)\end{array}$ & $\begin{array}{c}\text { Male } \\
\text { No }(\%)\end{array}$ & $\begin{array}{l}\text { Female } \\
\text { No }(\%)\end{array}$ & $\begin{array}{l}\text { Medical } \\
\text { No }(\%)\end{array}$ & $\begin{array}{c}\text { Non-medical } \\
\text { No }(\%)\end{array}$ & \\
\hline $\begin{array}{l}\text { Atherosclerosis increases } \\
\text { the risk of stroke }\end{array}$ & $\begin{array}{c}22 \\
(57.4)\end{array}$ & $\begin{array}{c}37 \\
(74)\end{array}$ & $\begin{array}{c}17 \\
(45)\end{array}$ & $\begin{array}{c}42 \\
(70)\end{array}$ & $\begin{array}{c}48 \\
(77.4)\end{array}$ & $\begin{array}{c}11 \\
(31.4)\end{array}$ & $\begin{array}{c}59 \\
(60.8)\end{array}$ \\
\hline $\begin{array}{l}\text { People with high blood } \\
\text { pressure are more liable } \\
\text { for atherosclerosis }\end{array}$ & $\begin{array}{c}21 \\
(44.7)\end{array}$ & $\begin{array}{r}43 \\
(86) \\
\end{array}$ & $\begin{array}{c}13 \\
(35.1)\end{array}$ & $\begin{array}{c}51 \\
(85)\end{array}$ & $\begin{array}{c}46 \\
(74.2)\end{array}$ & $\begin{array}{c}18 \\
(51.4)\end{array}$ & $\begin{array}{c}64 \\
(66)\end{array}$ \\
\hline $\begin{array}{l}\text { Arteriosclerosis can } \\
\text { cause renal damage }\end{array}$ & $\begin{array}{c}19 \\
(40.4)\end{array}$ & $\begin{array}{c}38 \\
(76)\end{array}$ & $\begin{array}{c}10 \\
(27)\end{array}$ & $\begin{array}{c}47 \\
(78.3)\end{array}$ & $\begin{array}{c}44 \\
(71)\end{array}$ & $\begin{array}{c}13 \\
(37.1)\end{array}$ & $\begin{array}{c}57 \\
(58.8)\end{array}$ \\
\hline $\begin{array}{l}\text { Atherosclerosis increases } \\
\text { the risk of thrombosis }\end{array}$ & $\begin{array}{c}30 \\
(63.8) \\
\end{array}$ & $\begin{array}{c}46 \\
(92) \\
\end{array}$ & $\begin{array}{c}26 \\
(70.3) \\
\end{array}$ & $\begin{array}{c}50 \\
(83.3) \\
\end{array}$ & $\begin{array}{c}57 \\
(92) \\
\end{array}$ & $\begin{array}{c}19 \\
(54.3) \\
\end{array}$ & $\begin{array}{c}76 \\
(78.4) \\
\end{array}$ \\
\hline $\begin{array}{l}\text { Medications can remove } \\
\text { the atherosclerotic } \\
\text { plaques from the vessel }\end{array}$ & $\begin{array}{c}15 \\
(31.9)\end{array}$ & $\begin{array}{r}32 \\
(64) \\
\end{array}$ & $\begin{array}{c}11 \\
(27.8)\end{array}$ & $\begin{array}{c}36 \\
(60) \\
\end{array}$ & $\begin{array}{c}40 \\
(64.5) \\
\end{array}$ & $\begin{array}{c}7 \\
(20) \\
\end{array}$ & $\begin{array}{c}47 \\
(48.5) \\
\end{array}$ \\
\hline $\begin{array}{l}\text { Chest and leg pains are } \\
\text { frequent symptoms of } \\
\text { atherosclerosis }\end{array}$ & $\begin{array}{c}19 \\
(40.4)\end{array}$ & $\begin{array}{c}36 \\
(72)\end{array}$ & $\begin{array}{c}12 \\
(32.4)\end{array}$ & $\begin{array}{c}43 \\
(71.7) \\
\end{array}$ & $\begin{array}{c}45 \\
(72.6)\end{array}$ & $\begin{array}{c}10 \\
(28.6)\end{array}$ & $\begin{array}{c}55 \\
(56.7)\end{array}$ \\
\hline $\begin{array}{l}\text { Diabetics are of great risk } \\
\text { of atherosclerosis }\end{array}$ & $\begin{array}{c}20 \\
(42.6)\end{array}$ & $\begin{array}{c}40 \\
(80)\end{array}$ & $\begin{array}{c}14 \\
(37.8)\end{array}$ & $\begin{array}{c}46 \\
(76.7)\end{array}$ & $\begin{array}{c}48 \\
(77.4)\end{array}$ & $\begin{array}{c}12 \\
(34.3)\end{array}$ & $\begin{array}{c}60 \\
(61.9)\end{array}$ \\
\hline $\begin{array}{l}\text { Chi-square } \\
\text { p value }\end{array}$ & & & & & & $\begin{array}{l}15.331 \\
0.0001^{*}\end{array}$ & \\
\hline
\end{tabular}

Awareness and knowledge of the students about stroke causes and complications:-

Seventy four students $(76.3 \%)$ could correctly recognize that hypertension is a major risk factor of stroke $(83.9 \%$ were of medical and $62.9 \%$ of non medical students). Less scores were obtained in the remaining questions in which only 63 students $(65 \%)$ were able to answer that Individuals with diabetes are more liable to suffer from stroke, 54 students $(55.7 \%)$ correctly realized that stroke is associated with difficulty in moving arms and legs, 68 students $(70.1 \%)$ knew that permanent speech defects are possible consequences of stroke, and 64 students answered correctly that stroke is often followed by memory disturbances $(66 \%)$. There was a significant statistical difference between awareness and knowledge of medical and non medical students $(\mathrm{p}=0.03)$, while no significant statistical difference was found between their age or gender and knowledge ( $\mathrm{p}=0.11$ and 0.61 respectively). Table 7

Table 7: Questions related to stroke knowledge answered correctly by the respondents

\begin{tabular}{|c|c|c|c|c|c|c|c|}
\hline & \multicolumn{2}{|c|}{ Age } & \multicolumn{2}{|c|}{ Gender } & \multicolumn{2}{|c|}{ Education } & \multirow{2}{*}{$\begin{array}{c}\text { Total } \\
\text { No } \\
(\%)\end{array}$} \\
\hline & $\begin{array}{c}16-20 \\
\text { No }(\%)\end{array}$ & $\begin{array}{c}21-25 \\
\text { No }(\%)\end{array}$ & $\begin{array}{c}\text { Male } \\
\text { No }(\%)\end{array}$ & $\begin{array}{l}\text { Female } \\
\text { No }(\%)\end{array}$ & $\begin{array}{c}\text { Medical } \\
\text { No }(\%)\end{array}$ & $\begin{array}{c}\text { Non-medical } \\
\text { No }(\%)\end{array}$ & \\
\hline $\begin{array}{l}\text { Individuals with diabetes } \\
\text { are more liable to suffer } \\
\text { from stroke }\end{array}$ & $\begin{array}{c}27 \\
(57.4) \\
\end{array}$ & $\begin{array}{c}36 \\
(72) \\
\end{array}$ & $\begin{array}{c}24 \\
(64.9) \\
\end{array}$ & $\begin{array}{r}39 \\
(65) \\
\end{array}$ & $\begin{array}{c}44 \\
(71) \\
\end{array}$ & $\begin{array}{c}19 \\
(54.3) \\
\end{array}$ & $\begin{array}{l}63 \\
(65) \\
\end{array}$ \\
\hline $\begin{array}{l}\text { Stroke is associated with } \\
\text { difficulty in moving arms } \\
\text { and legs }\end{array}$ & $\begin{array}{c}20 \\
(42.6) \\
\end{array}$ & $\begin{array}{c}34 \\
(68) \\
\end{array}$ & $\begin{array}{c}22 \\
(59.4) \\
\end{array}$ & $\begin{array}{c}32 \\
(53.3) \\
\end{array}$ & $\begin{array}{c}40 \\
(64.5) \\
\end{array}$ & $\begin{array}{c}14 \\
(40) \\
\end{array}$ & $\begin{array}{c}54 \\
(55.7) \\
\end{array}$ \\
\hline $\begin{array}{l}\text { Permanent speech defects } \\
\text { are possible consequences } \\
\text { of stroke }\end{array}$ & $\begin{array}{c}30 \\
(63.8) \\
\end{array}$ & $\begin{array}{c}38 \\
(76) \\
\end{array}$ & $\begin{array}{c}29 \\
(78.4) \\
\end{array}$ & $\begin{array}{c}39 \\
(65) \\
\end{array}$ & $\begin{array}{c}49 \\
(79) \\
\end{array}$ & $\begin{array}{c}19 \\
(54.3) \\
\end{array}$ & $\begin{array}{c}68 \\
(70.1) \\
\end{array}$ \\
\hline $\begin{array}{l}\text { A stroke is often followed } \\
\text { by memory disturbances }\end{array}$ & $\begin{array}{c}28 \\
(59.6) \\
\end{array}$ & $\begin{array}{c}36 \\
(72) \\
\end{array}$ & $\begin{array}{c}25 \\
(67.6) \\
\end{array}$ & $\begin{array}{c}39 \\
(65) \\
\end{array}$ & $\begin{array}{c}48 \\
(77.4) \\
\end{array}$ & $\begin{array}{c}16 \\
(45.7) \\
\end{array}$ & $\begin{array}{c}64 \\
(66) \\
\end{array}$ \\
\hline $\begin{array}{l}\text { Hypertension is a major } \\
\text { risk factor of stroke }\end{array}$ & $\begin{array}{c}30 \\
(63.8)\end{array}$ & $\begin{array}{c}44 \\
(88)\end{array}$ & $\begin{array}{c}27 \\
(73)\end{array}$ & $\begin{array}{c}47 \\
(78.3)\end{array}$ & $\begin{array}{c}52 \\
(83.9)\end{array}$ & $\begin{array}{c}22 \\
(62.9)\end{array}$ & $\begin{array}{c}74 \\
(76.3)\end{array}$ \\
\hline $\begin{array}{l}\text { Chi-square } \\
\mathrm{P} \text { value }\end{array}$ & \multicolumn{2}{|c|}{$\begin{array}{c}3.963 \\
0.11\end{array}$} & \multicolumn{2}{|c|}{$\begin{array}{c}0.545 \\
0.61\end{array}$} & \multicolumn{3}{|c|}{$\begin{array}{l}6.039 \\
0.03^{*}\end{array}$} \\
\hline
\end{tabular}




\section{Discussion:-}

Metabolic syndrome is a worldwide public health problem and its prevalence is constantly increasing [14]. The presence of metabolic syndrome is associated with increased the risk for both atherosclerotic cardiovascular disease and type 2 diabetes mellitus and therefore it should be addressed as an important clinical issue. While there are several definitions nevertheless, all of them include the following factors: Abdominal obesity, elevated blood pressure, high triglycerides, low high-density cholesterol (HDL cholesterol) and signs of insulin resistance, such as high fasting plasma glucose level [3].

The aim of this study is to assess knowledge and awareness of conditions relevant to MS namely diabetes mellitus, hypertension, obesity, serum cholesterol level atherosclerosis and stroke, among a sample of college students in the University of Tabuk.

Regarding students' knowledge about diabetes, it was found that more than half of the total sample of students correctly reported that there are several types of diabetes $(85.6 \%)$, diabetes can cause eye disorders (65\%), frequent urination $(86.6 \%)$ and poor appetite $(54.6 \%)$, while less than half of the students correctly answered that hereditary factors play a major role in development of all types of diabetes (43.3\%). A statistical significant relationship was found between students' knowledge and their education major (medical versus non-medical students) ( $\mathrm{p}=0.04$ ), whereas, no statistical significant difference was found between students' knowledge and their age or gender $(\mathrm{p}=0.22$ and 0.5 respectively).

When analyzing students' answers for hypertension symptoms, complications and treatment related questions, it was observed that more than half of the students have good knowledge about hypertension this may be attributed to hypertension is a common health problem and most of students are familiar with its symptom, complications and treatment. Most of the correct answers was found between medical students and older students age group with statistical significant difference ( $\mathrm{p}=0.04$ and 0.03 respectively).

The same finding was observed regarding the students' knowledge about obesity risk, complications and prevention. As the majority of them could correctly answered the obesity related questions. No statistical significant difference was found between students' knowledge and their age $(\mathrm{p}=0.16)$, gender $(\mathrm{p}=0.34)$ or their education major $(\mathrm{p}=0.31)$ this may be due to that knowledge about obesity related conditions are well known among most of students whether medical or non-medical as many students are usually concerned about their body shapes.

Also, in the present study, it was found that most of the students realized that high cholesterol level can cause atherosclerosis (74.2\%), not treated with medications only (73.2\%) and diabetes is associated with high cholesterol level. Most of the correct answers was detected among medical students with statistical significant difference $(\mathrm{p}=0.02)$. While, no statistical significant difference was found between students' knowledge and their age $(\mathrm{p}=0.319)$ or gender $(\mathrm{p}=0.41)$.

Regarding students' knowledge and awareness about atherosclerosis symptoms, complications and treatment, a fair good understanding of atherosclerosis related conditions was significantly found among medical rather than nonmedical students $(\mathrm{p}=0.0001)$, in older age group students more than younger age $9 \mathrm{p}=0.002)$ and in females more than males $(\mathrm{p}=0.0001)$.

Finally we studied the college students' knowledge about stroke related conditions, and it was found that more than half of students are aware of stroke symptoms and causes. There was a statistical significant difference between students' knowledge and their major $(\mathrm{p}=0.03)$, on the other hand no statistical significant relation was found between students' knowledge and their age $(\mathrm{p}=0.11)$ or gender $(\mathrm{p}=0.61)$.

Findings from this study suggest that type of education (medical versus non-medical college) may be a significant factor in awareness and knowledge of MS relevant conditions.

Although not reaching statistical significance, younger students aged 16-20 years were less likely than older students 21-25 years to identify diabetes mellitus, obesity, serum cholesterol level, atherosclerosis and stroke manifestations and complications; this was the same in relation to gender as female students were able to recognize the MS related conditions more adequately than male students. One possible explanation for this difference in results might be that female students are more interest in health issues than male students at college age, or may be due to that male students, in general, have more interest in extracurricular activities than in health issues. 
It was found that, the majority of students were most knowledgeable about most of diabetes mellitus, hypertension, obesity and cholesterol serum level associated manifestations and complications and least and knowledgeable about atherosclerosis and stroke. In spite of their good knowledge about diabetes, hypertension and obesity, there were some misconceptions acquired by students on some questions especially among younger age group and non-medical college students, this may most probably due to media like television magazines, and internet and how they could affect most college students' health knowledge consequently this will have implications for future health education efforts to correct the misconceptions among college students about major health problems.

The results of this study recommended that health education programs is need to designed and targets the nonmedical college students to improve their knowledge and awareness. Also, educating college students about cardiovascular diseases and stroke is very important; especially risk factors for heart disease that can occur at an early age. Colleges especially non-medical ones should emphasize not only on students' course curriculum teaching but also on students' health education to promote good health and minimize the development of MS risk factors among students at an early age.

\section{Conclusion:-}

Students' knowledge and awareness of conditions relevant to metabolic syndrome differed among medical and nonmedical students, with few age group and gender differences and few false beliefs. Thus, educating students about this disease as a first step in awareness and knowledge is important method that could lead to actual behavior changes to reduce their personal risk of developing diabetes or metabolic syndrome later in life.

\section{Conflict of interest:-}

The authors have no conflicts of interest to report.

\section{References:-}

1. Alberti K, Eckel RH, Grundy SM et al. "Harmonizing the metabolic syndrome: a joint interim statement of the international diabetes federation task force on epidemiology and prevention; National heart, lung, and blood institute; American heart association; World heart federation; International atherosclerosis society; And international association for the study of obesity," Circulation 2009; 120 (16): 1640-1645.

2. Yahia N, Brown C, Rapley M, Chung M. Assessment of college students' awareness and knowledge about conditions relevant to metabolic syndrome . Diabetology \& Metabolic Syndrome 2014; 6:111-126.

3. Grundy SM, 1Brewer 1James HB, Cleeman I, 1Smith SC,Lenfant C. Definition of Metabolic Syndrome: Report of the National Heart, Lung, and Blood Institute/American Heart Association Conference on Scientific Issues Related to Definition. Circulation.2004;109: 433-438.

4. Reaven GM: Role of insulin resistance in human disease. Diabetes 1988; 37(13):1595-1607.

5. Desroches S, Lamarche B. The evolving definitions and increasing prevalence of the metabolic syndrome. Applied Physiology Nutrition and Metabolism 2007; 32(1): 23-32.

6. Kolovou GD, Anagnostopoulou KK, Salpea KD, Mikhailidis DP. The prevalence of metabolic syndrome in various populations. American Journal of the Medical Sciences 2007; 333(6): 362-371.

7. International Diabetes Federation: The IDF consensus worldwide definition of the metabolic syndrome.

8. Al-Lawati JA, Mohammad AJ, Al-Hinal HQ, Jousilahti P: Prevalence of the metabolic syndrome among Omani adults. Diabetes Care 2003; 26:1781-1785.

9. Abdul-Rahim HF, Husseini A, Bjertness E, Giacaman R, Gordon NH, Jervell J. The metabolic syndrome in the West Bank population. An urban-rural comparison. Diabetes Care 2001; 24: 275-279.

10. Jaber LA, Brown MB, Hammad A, Zhu Q, Herman WH. The prevalence of the metabolic syndrome among Arab Americans. Diabetes Care 2004;27: 234-8.

11. Barrimah IE, Mohaimeed AR, Midhat F, Al-Shobili HA. Prevalence of Metabolic Syndrome Among Qassim University Personnel in Saudi Arabia. Int J Health Sci. (Qassim) 2009; 3:133-142.

12. Meigs JB, Wilson PW, Fox CS, Vasan RS, Nathan DM, Sullivan LM, D'Agostino RB: Body mass index, metabolic syndrome, and risk of type 2 diabetes or cardiovascular disease. J Clin Endocrinol Metab 2006; 91(8):2906-2912.

13. Alberti KG, Zimmet P. The metabolic syndrome - a new worldwide definition. The Lancet, 2005; 366 (9491):10591062.

14. Lim S, Shin H, Song JH, et al. Increasing prevalence of metabolic syndrome in Korea: The Korean National Health and Nutrition Examination Survey for 1998-2007. Diabetes Care 2011; 34(6): 1323-1328. 